\title{
How do interplanetary shock impact angles control the size of the geoeffective magnetosphere?
}

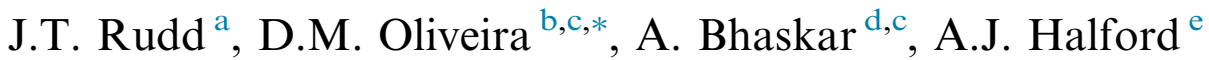 \\ ${ }^{a}$ Department of Physics, United States Naval Academy, Annapolis, MD, USA \\ ${ }^{\mathrm{b}}$ Goddard Planetary Heliophysics Institute, University of Maryland Baltimore County, Baltimore, MD, USA \\ ${ }^{\mathrm{c}}$ NASA Goddard Space Flight Center, Greenbelt, MD, USA \\ ${ }^{\mathrm{d}}$ University Corporation for Atmospheric Research, Boulder, CO, USA \\ ${ }^{\mathrm{e}}$ The Aerospace Corporation, Chantilly, VA, USA
}

Received 3 May 2018; received in revised form 7 September 2018; accepted 9 September 2018

\begin{abstract}
In this paper, we investigate temporal and spatial magnetosphere response to the impact of interplanetary (IP) shocks with different inclinations and speeds on the Earth's magnetosphere. A data set with more than 500 IP shocks is used to identify positive sudden impulse $\left(\mathrm{SI}^{+}\right)$events as expressed by the SuperMAG partial ring current index. The $\mathrm{SI}^{+}$rise time (RT), defined as the time interval between compression onset and maximum $\mathrm{SI}^{+}$signature, is obtained for each event. We use RT and a model suggested by Takeuchi et al. (2002) to calculate the geoeffective magnetospheric distance (GMD) in the shock propagation direction as a function of shock impact angle and speed for each event. GMD is a generalization of the geoeffective magnetosphere length (GML) suggested by Takeuchi et al. (2002), defined from the subsolar point along the X line toward the tail. We estimate statistical GMD and GML values which are then reported for the first time. We also show that, similarly to well-known results for RT, the highest correlation coefficient for the GMD and impact angle is found for shocks with high speeds and small impact angles, and the faster and more frontal the shock, the smaller the GMD. This result indicates that the magnetospheric response depends heavily on shock impact angle. With these results, we argue that the prediction and forecasting of space weather events, such as those caused by coronal mass ejections, will not be accurately accomplished if the disturbances' angles of impact are not considered as an important parameter within model and observation scheme capabilities.
\end{abstract}

(C) 2018 COSPAR. Published by Elsevier Ltd. All rights reserved.

Keywords: Interplanetary shocks; Shock impact angle; Geomagnetic activity; Geoeffective magnetospheric distance

\section{Introduction}

Interplanetary (IP) shocks correspond to a key phenomenon of Space Weather investigations. As they impact Earth, geomagnetic disturbances can be detected on and in the ground, and in the geospace. Examples are auroral substorms, ionospheric total electron content, intensification

\footnotetext{
* Corresponding author at: Goddard Planetary Heliophysics Institute, University of Maryland Baltimore County, Baltimore, MD, USA.

E-mail address: denny.m.deoliveira@nasa.gov (D.M. Oliveira).

and dynamics of field-aligned currents connecting the ionosphere-magnetosphere system, heating of neutral particles in the high-latitude regions of the upper atmosphere, enhancement of geomagnetically induced currents that may lead to power distribution interruption, and variations of particle populations and energy levels in the radiation belt. Several aspects of IP shock geoeffectiveness can be found elsewhere (see, e.g., Alves et al., 2011; Tsurutani et al., 2011; Singh et al., 2017; Oliveira and Samsonov, 2018; Oliveira et al., 2018). 
On the ground, the first dramatic magnetosphere response to IP shock impacts is generally characterized by a step-like increase in the horizontal geomagnetic field whose signature is named sudden impulse, positive $\left(\mathrm{SI}^{+}\right)$ or negative $\left(\mathrm{SI}^{-}\right)$for magnetospheric compressions and expansions, respectively (Nishida and Jacobs, 1962; Araki, 1977, 1994). Early observations such as reported by Dessler et al. (1960) and Ondoh (1963) defined the SI ${ }^{+}$ rise time (RT) as the time interval between the compression onset and the time of maximum perturbation of the horizontal magnetic field on the ground. Nishida (1966) concluded that several aspects of IP shocks may play important roles in determining $\mathrm{SI}^{+}$RTs, such as shock thickness, IP shock RT, speed, and the time the shock or discontinuity front takes to sweep over the magnetosphere. Kaufmann and Konradi (1969) suggested that the shock "geoeffective" time can be increased by an IP shock impact on either dawn or dusk flanks as a result of an asymmetric compression. In general, early works concluded that shockassociated $\mathrm{SI}^{+}$RTs depend heavily on the time shocks take to cover the geoeffective magnetospheric distance, but the association between shock impact angle and $\mathrm{SI}^{+} \mathrm{RT}$ was addressed much later, as will be discussed further in this paper.

The IP shock impact angle, defined as the angle between the shock normal vector and the Sun-Earth line, has recently shown to be an important feature of IP shock geoeffectiveness. Samsonov (2011) predicted that a highly inclined shock whose normal lay in the equatorial plane would drive a strong downstream solar wind velocity $v_{y}$ component, increasing the dynamic pressure in the dawndusk direction, which would compress the magnetosphere asymmetrically and trigger an overshoot in the magnetosphere flank opposite to the flank of shock impact. This prediction was later confirmed with simulations and observations by Samsonov et al. (2015). With simulations, Oliveira and Raeder (2014) showed that a moderate frontal shock can be more geoeffective than a strong and inclined shock due to symmetric compressions, as represented by magnetic field enhancements, as well as ionospheric currents and auroral precipitation energy intensifications. These results were later confirmed by Oliveira and Raeder (2015) with geomagnetic data provided by a large number of ground stations, where, most generally, the faster and the more frontal the shock, the higher the shock geoefficiency, as represented by auroral substorms. Similar results were obtained for nightside auroral power intensifications by Oliveira et al. (2016). The main conclusion of these efforts corresponds to the fact that almost symmetric compressions create highly favorable scenarios for the maximization of geomagnetic activity triggered by impacts of IP shocks with small inclinations on the Earth's magnetosphere. A comprehensive review of this topic has recently been provided by Oliveira and Samsonov (2018).

The main goal of this paper is to explore the spatial and temporal effects associated with $\mathrm{SI}^{+}$events caused by the impact of IP shocks with different inclinations on the magnetosphere. We use a data set with over 500 IP shocks detected at $1 \mathrm{AU}$ in a time range of more than two decades to determine, for the first time, average distances of the magnetosphere when compressed by IP shocks with different impact angles and speeds. We find that the geoeffective magnetospheric distances parallel to the shock propagation direction correlate well with shock impact angles. In general, we find that the faster and the more frontal the shock, the shorter the geoeffective magnetospheric distances. This indicates that inclined shocks spend more time in the magnetosphere and slowly compress it, as opposed to frontal shocks. These results suggest that modelers and experimentalists should take into consideration the angles with which solar disturbances strike the Earth's magnetosphere in their space weather prediction tools to improve their prediction and forecasting capabilities.

\section{Dataset}

\subsection{Interplanetary shock database}

In this paper, we use an extended version of the IP shock database published by Oliveira and Raeder (2015) to investigate the effects of shock impact angles on ground magnetometer responses as represented by $\mathrm{SI}^{+}$events. This shock list currently contains 547 fast forward shocks observed by both Wind and ACE (Advanced Composition Explorer) spacecraft at the L1 point in the time period of January 1995 to September 2017. IP shock parameters, such as shock impact angle, speed, compression ratio, amongst others, were calculated by using standard shock theory, namely the Rankine-Hugoniot jump conditions, with the assumptions of energy and momentum conservation through the shock surface (see, e.g., Landau and Lifshitz, 1960). The interplanetary magnetic field (IMF) and plasma data recorded by Wind and ACE were used in the computation of the shock properties. For a detailed description of the parameter calculations related to this work, we refer the works of Oliveira and Raeder (2015) and Oliveira (2017), as well as a recent review by Oliveira and Samsonov (2018).

The shock impact angle, here represented by $\theta_{x_{n}}$, corresponds to the angle between the shock normal vector and the geocentric solar ecliptic (GSE) X line. With this choice, a shock with $\theta_{x_{n}}=180^{\circ}$ indicates a purely frontal shock whose shock normal aligns with the $\mathrm{X}$ line, and points toward Earth. Shocks then become inclined if $\theta_{x_{n}}$ decreases. The shock speed $v_{s}$ is measured relatively to the spacecraft or Earth.

\subsection{SuperMAG ground magnetometer data}

A ground magnetometer network enables to study the global and average magnetospheric response to IP shock impacts. The ground magnetometer response is here represented by the enhanced SuperMAG partial ring current 
index SMR introduced by Newell and Gjerloev (2012). This index is part of a series of enhanced geomagnetic indices derived by the SuperMAG initiative that involves worldwide ground magnetometer arrays (see, e.g Gjerloev, 2009; Newell and Gjerloev, 2012). The use of these indices is justified by the idea that the probability of measuring overhead current systems increases with the number of magnetometers right below on the ground. As opposed to the traditional SYM-H index, currently computed by 6 stations in mid- and low-latitude regions, the SMR index is computed by approximately 100 stations in the same regions. Although the SMR index is very similar in essence to the SYM-H index, Newell and Gjerloev (2012) showed that the former presents large local time differences during different geomagnetic storm phases. The time resolution of the SMR index is the same as the SYM-H index, $1 \mathrm{~min}$. SuperMAG index data can be downloaded from the SuperMAG initiative website located at http://supermag.jhuapl.edu.

\section{Role of shock impact angle in the spatial/temporal magnetosphere response}

Fig. 1 shows solar wind dynamic pressure $\left(P_{d y}=\rho v^{2}\right)$ and the SMR index for two distinct IP shocks. The left column shows results for the highly inclined shock (HIS) of 01 May 1997, while the right column shows results for the nearly frontal shock (NFS) of 25 March 2014. HIS shows impact angle $\theta_{x_{n}}=133.63^{\circ}$, shock speed $v_{s}=380.03$ $\mathrm{km} \mathrm{s}^{-1}$, and magnetosonic Mach number $M_{s}=2.1$. The same quantities for NFS are $\theta_{x_{n}}=176.97^{\circ}, v_{s}=386.60 \mathrm{~km}$ $\mathrm{s}^{-1}$, and $M_{s}=2.3$. Therefore, both shocks have similar strengths (approximately the same shock speeds and Mach numbers). Wind data were used for the parameter calculations of both shocks. The solar wind data were shifted to the bow shock to match in time with the magnetosphere response as indicated by SMR.

In all panels, the first dashed vertical line indicates the compression onset, or the moment in which SMR rises characterizing an $\mathrm{SI}^{+}$signature. Fig. 1a shows that the HIS compression lasts for $8 \mathrm{~min}$, when $P_{d y}$ reaches its maximum value. The SMR index (Fig. 1c) still continues to increase for 2 more minutes, which indicates a late magnetospheric response. The rise time (RT) in this case is $10 \mathrm{~min}$, as represented by the shaded green and blue areas. Although generally shock RTs in the solar wind are very sharp, there is a slight difference between such RTs for the HIS and the NFS, with the former being $\sim 2$ min longer than the latter. The SMR amplitude in response to the HIS, as indicated by $\triangle S M R$, is $16.3 \mathrm{nT}$. In the case of the NFS, Fig. $1 \mathrm{~b}$, the instance of time with maximum pressure $P_{d y}$ matches with the instance of time with maximum SMR (Fig. 1d). In this case, $\mathrm{RT}=5 \mathrm{~min}$, and $\triangle S M R=26.6 \mathrm{nT}$. This shows a prompt magnetosphere response to the
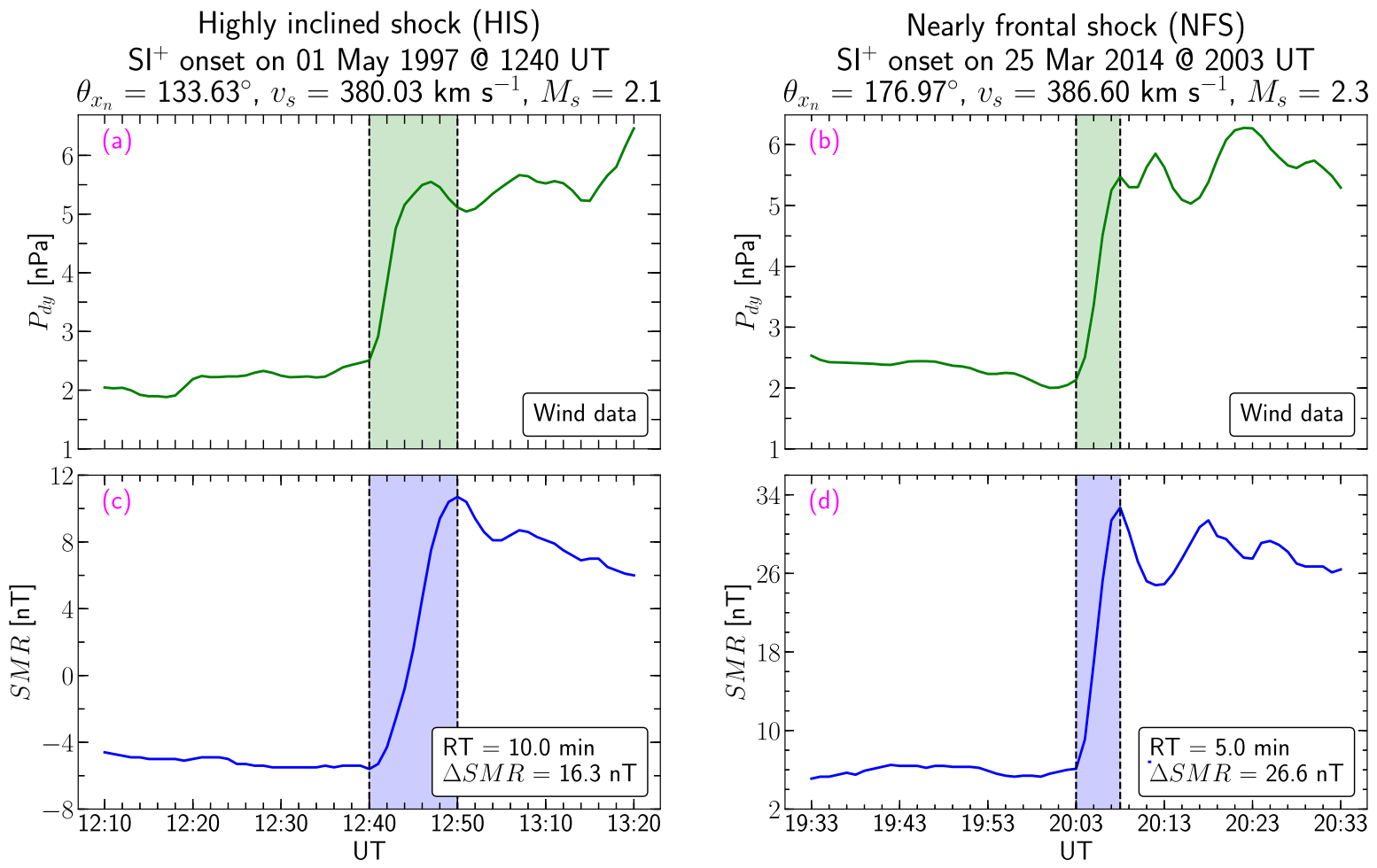

Fig. 1. Examples of two fast forward IP shocks with similar strengths and different impact angles observed by Wind at the L1 point upstream of the Earth. Highly inclined shock (HIS, $\theta_{x_{n}}=133.63^{\circ}$ ), dynamic pressure (a) and SMR (c). Nearly frontal shock (NFS, $\theta_{x_{n}}=176.97^{\circ}$ ), dynamic pressure (b) and SMR (d). The shaded areas for the HIS show RT $=10 \mathrm{~min}$, while the shaded areas for NFS show RT $=5$ min. 
impact of the NFS and its subsequent compression of the magnetosphere.

As suggested by Takeuchi et al. (2002), the large RT for the HIS here represented is explained by the fact that the shock had a large impact angle. This in turn triggers a longer magnetosphere response, since the shock takes more time to sweep over the magnetosphere. In contrast, the NFS triggers a shorter RT because of its small impact angle. The shock then travels a shorter distance in the magnetosphere during compression, compressing the most important current systems close to Earth (mainly the dayside magnetopause current) in a shorter time interval leading to a faster and stronger geoeffective magnetospheric response. The importance of the shock impact angle is also reflected on the SMR amplitude response since its contributions come mostly from the magnetopause current variations.

In order to estimate the approximate magnetospheric distance traveled by the shocks while the magnetosphere is effectively being compressed, we use the simple model suggested by Takeuchi et al. (2002). In that model, the shock impact angle is taken into account in the distance computations because this distance is calculated along the direction of the shock propagation tail-ward from the first point of contact between the shock and the magnetopause. This model is based on an assumption suggested by Nishida (1966) who relates the RT occurrence with the time taken by a discontinuity or IP shock front to sweep by the geoeffective distance along the magnetosphere. Although other effects discussed by Nishida (1966) may influence $\mathrm{SI}^{+} \mathrm{RTs}$, here they are considered secondary and disregarded (see additional discussion by Araki et al., 2004). However, early studies predicted disturbance propagation speeds in the magnetosphere in the velocity range 450 $1500 \mathrm{~km} \mathrm{~s}^{-1}$ (Nopper et al., 1982; Wilken et al., 1977). In fact, observation and simulation results reported by Andréeová et al. (2008) confirmed these predictions, where disturbance speeds were usually higher than the shock speed in the solar wind. Such differences were smaller in the dayside magnetosphere, and larger in the nightside magnetosphere increasing along the tail. Since the most significant contributions to $\mathrm{SI}^{+}$event formation, and its consequent RT, come from current systems located in the dayside near Earth, as opposed to nightside magnetotail currents, we assume, at least in a first approximation, the shock speed to be invariant while the shock compresses the magnetosphere. Therefore, we define this distance as the geoeffective magnetosphere distance (GMD), as a function of RT and $v_{s}$, by the simple relationship

$\mathrm{GMD}=v_{s} \times \mathrm{RT}$,

where GMD is given in Earth's radii, with $R_{E}=6371 \mathrm{~km}$.

In addition, another important distance, suggested by Takeuchi et al. (2002), the geoeffective magnetopause length (GML), defined as the GMD component parallel to the GSE $\mathrm{X}$ line between the first shock/magnetopause point of contact and the farther magnetopause boundary, is given by:

$\mathrm{GML}=\left[v_{s} \times \mathrm{RT}\right]\left|\cos \left(\theta_{x_{n}}\right)\right|$.

Both the GMD and GML mean values will be statistically estimated in this paper for the first time. The relationship between GMD/GML and shock speed and correlations with impact angle will also be determined.

The geometric aspects of the magnetosphere while being compressed by both HIS and NFS as determined by the Takeuchi et al. (2002) model are shown in Fig. 2 for the HIS, upper panel, and NFS, lower panel. The dashed and continuous black lines indicate magnetosphere configurations of the quiet and compressed magnetosphere according to the Shue et al. (1998) model, respectively. This model determines the magnetopause positions as a function of solar wind $\left(P_{d y}\right)$ and IMF $\left(B_{z}\right)$ conditions. The IMF $B_{z}$ and $P_{d y}$ values shown in Fig. 2 correspond to averaged downstream values. In both panels, the first green line indicates the shock front at the moment of first contact with the magnetosphere, while the second green line indicates the shock front position at the end of the $\mathrm{SI}^{+}$event, whose RTs are indicated in Fig. 1. The thick red ${ }^{1}$ arrows in both panels indicate the respective shock normal directions. The red line connecting both shock fronts corresponds to the GMD, whereas the horizontal blue line indicates the GML position. The dashed vertical blue line shows the average magnetosphere width $w_{1}$ of the quiet magnetosphere, while the continuous blue line superposed to $w_{1}$ indicates the average magnetosphere width of the compressed magnetosphere.

The quiet magnetosphere configurations before the HIS and NFS impacts are very similar. Due to their shock impact angles, the HIS hits the magnetosphere in the post-dawn sector, while the NFS hits the magnetosphere almost at the subsolar point. Since both shocks have similar strengths, the magnetosphere width is reduced by approximately $60 \%$ in both cases. Given RT and $v_{s}$ for each shock, the model yields $\mathrm{GMD}=36.73 R_{E}$ and $\mathrm{GML}=25.34 R_{E}$ for the HIS, and GMD $=18.20 R_{E}$ and $\mathrm{GML}=18.20 R_{E}$ for the NFS. The GMD associated with the HIS is twice larger as the GMD associated with the NFS, even though both shocks have similar speeds. The HIS-associated GML is $\sim 70 \%$ the size of the HISassociated GMD, while in the case of the NFS both distances are almost the same because the NFS has a very small inclination. Such geoeffective magnetosphere configurations explain the differences in the RTs observed in the bottom row of Fig. 1.

These results clearly show that the shock impact angle plays an important role in determining the compression time and size of the geoeffective magnetosphere. In the next section we will compute correlations between GMD(GML) and shock impact angle.

\footnotetext{
${ }^{1}$ For interpretation of color in Fig. 1, the reader is referred to the web version of this article.
} 

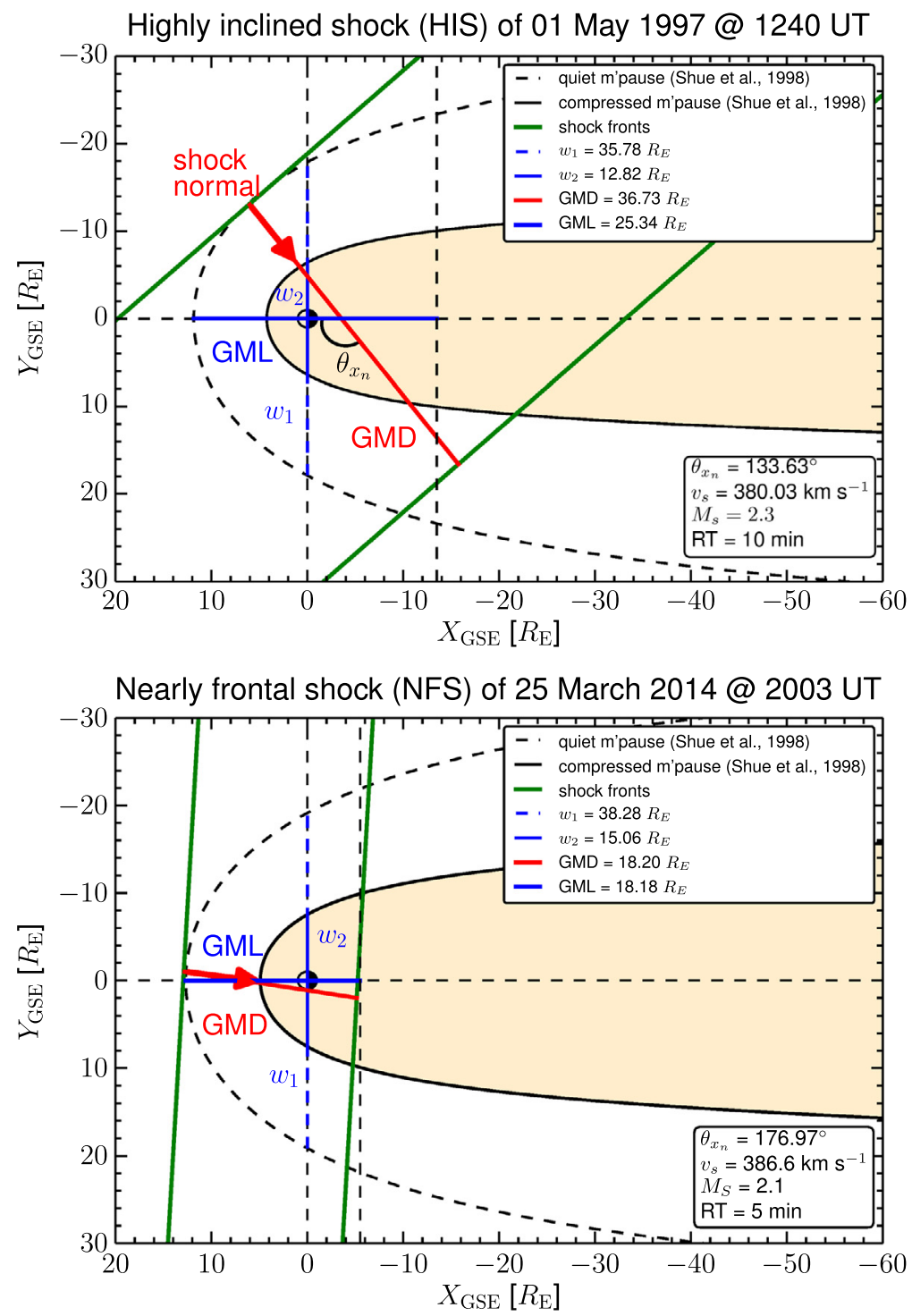

Fig. 2. An illustration of the simple model for the determination of geoeffective magnetospheric distances accounting for shock impact angle suggested by Takeuchi et al. (2002). The model is applied to the highly inclined shock (HIS, upper panel) and the nearly frontal shock (NFS, lower panel). Both shock properties are shown in Fig. 1. The compressed magnetopause limits are obtained by the Shue et al. (1998) model. The red thick arrows indicate each shock normals and their respective points of first interaction with the magnetopause. Shock properties and RTs used for each shock are shown in each panel, as well as the calculated distances GMD, GML and magnetosphere widths. (For interpretation of the references to colour in this figure legend, the reader is referred to the web version of this article.)

\section{Statistical results}

\subsection{General IP shock properties}

Fig. 3 shows statistical results for the yearly shock distribution and the shock parameters used in the model computations, as well as the subsequent magnetospheric temporal and spatial responses. Fig. 3a shows yearly IP shock number (cyan bars), while the continuous black line indicates the monthly-averaged SunSpot Number (SSN), obtained from the World Data Center Sunspot Index and Longterm Solar Observations (WDC-SILO), Royal Observatory of Belgium, Brussels (http://www.sidc.be/silso/datafiles). The time span covers the end of the declining phase of
Solar Cycle (SC) 22, the entire SC23, and almost all SC24. In general, Fig. 3a shows that the yearly IP shock numbers follow the SSN, being more numerous when SSNs are large. Since the current SC has shown the lowest solar activity during the space era (Pesnell, 2015), the number of shocks in SC24 are comparatively small.

Results for shock speed (Fig. 3b) show that average shock speed at $1 \mathrm{AU}$ is $\sim 470 \mathrm{~km} \mathrm{~s}^{-1}$. The lower quartile (LQ) and upper quartile (UQ) for shock speed are $\sim 360 \mathrm{~km} \mathrm{~s}^{-1}$ and $\sim 550 \mathrm{~km} \mathrm{~s}^{-1}$, respectively. The RT, shown in Fig. 3c, shows a mean value of $\langle\mathrm{RT}\rangle=\sim 6.6 \mathrm{~min}$ and $5.0 \mathrm{~min}$ and $8.0 \mathrm{~min}$ for both quartiles, respectively. The shock impact angle $\theta_{x_{n}}$ (Fig. 3d) shows a mean value of $\sim 150^{\circ}$, while LQ and UQ results are $\sim 137^{\circ}$ and 


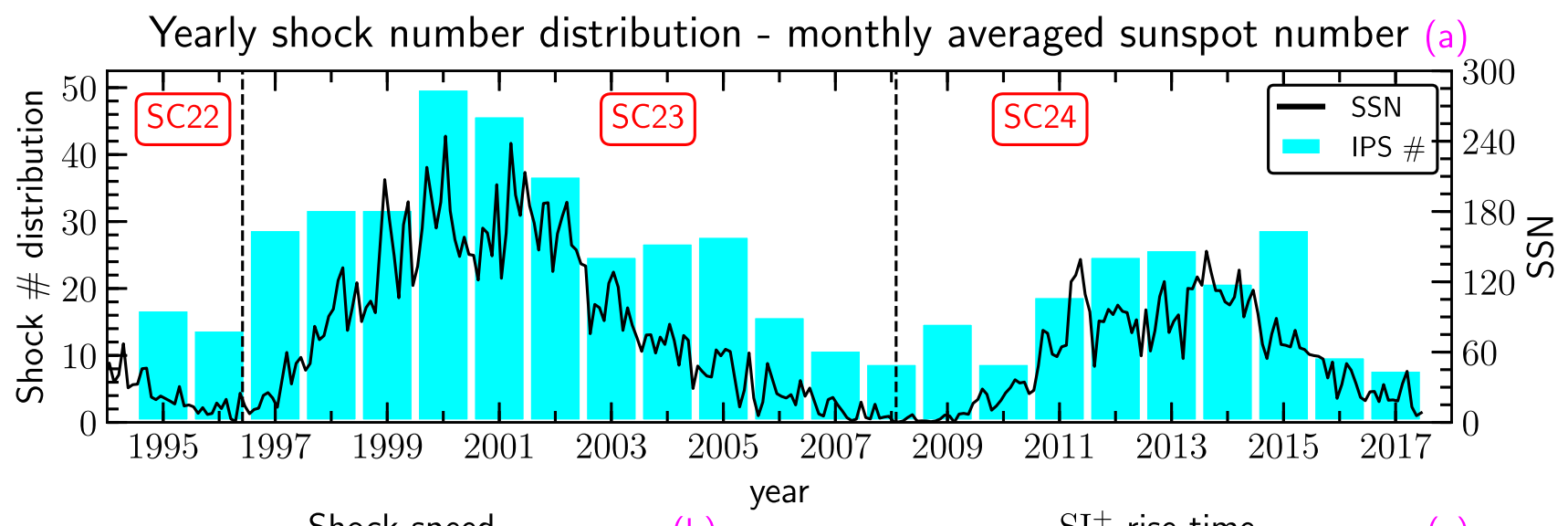

Shock speed

(b)
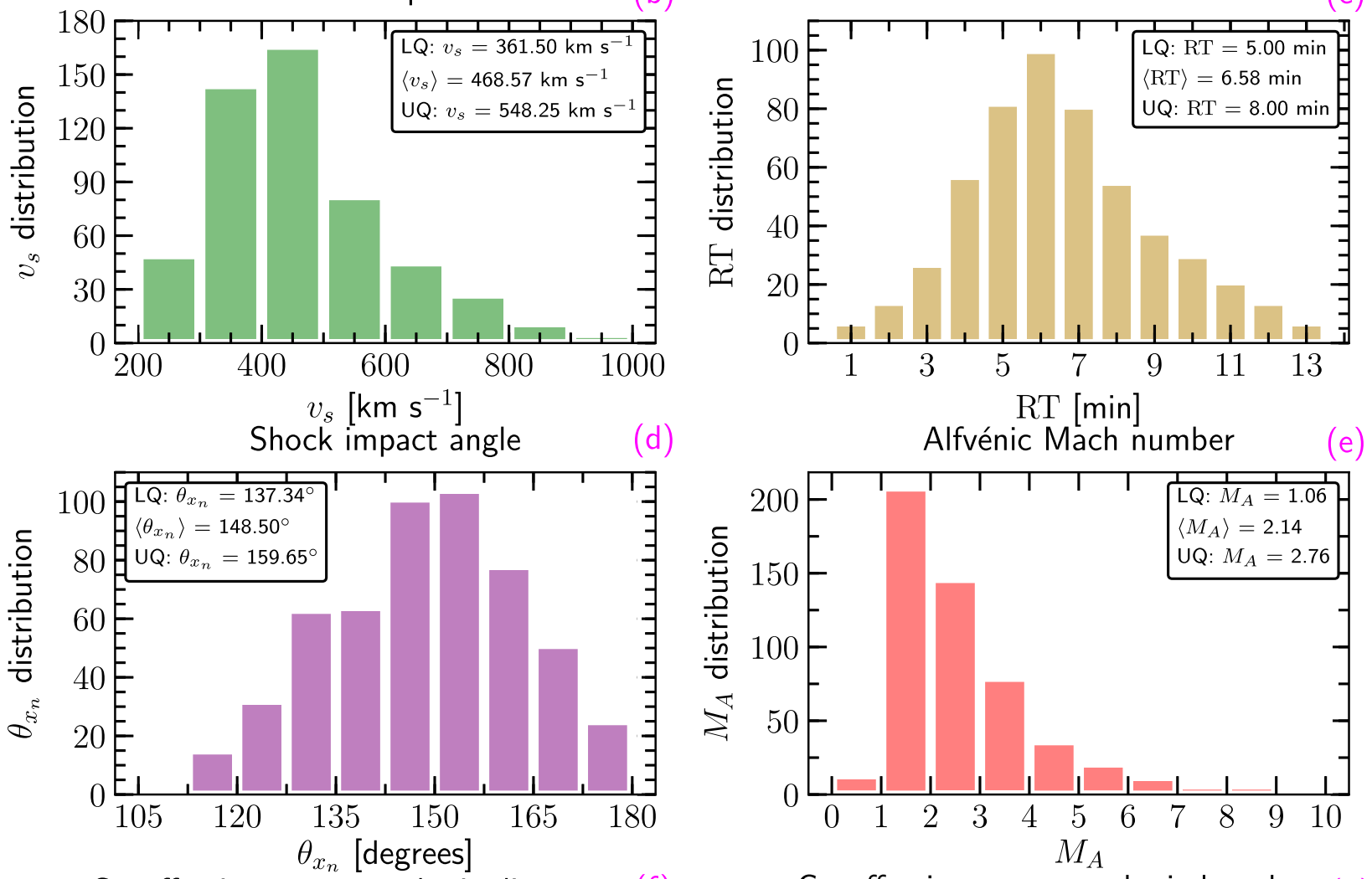

Alfvénic Mach number

(e)

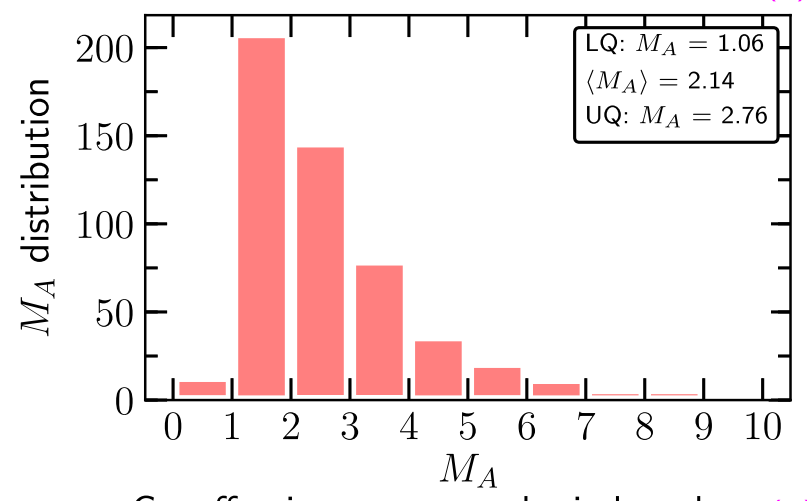

Geoeffective magnetospheric distance (f)

Geoeffective magnetospheric length
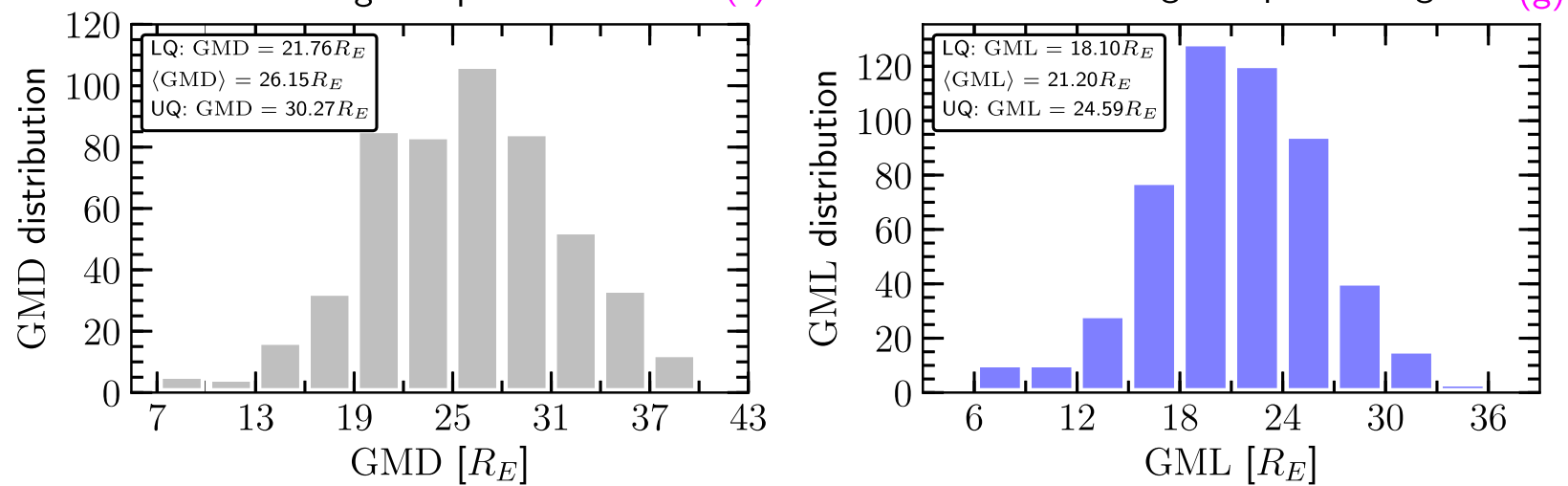

Fig. 3. Statistical distributions of IP shock properties, $\mathrm{SI}^{+} \mathrm{RTs}$, and geoeffective magnetospheric distances obtained in this study. Yearly shock number distribution and monthly-averaged SSN (a), with the dashed vertical lines indicating the limits of the solar cycles; shock speed $v_{s}$ (b); rise time RT (c); shock impact angle $\theta_{x_{n}}$ (d); Alfvénic Mach number $M_{A}$ (e); geoeffective magnetosphere distance (f); and geoeffective magnetosphere length (GML). (b-g) show statistical values for lower quartile (LQ), average $(\langle\mathrm{X}\rangle)$, and upper quartile for each quantity $\mathrm{X}$. 
$\sim 160^{\circ}$, respectively. These shock properties have already been discussed before (Oliveira and Raeder, 2015; Kilpua et al., 2015; Oliveira and Samsonov, 2018). For GMD (Fig. 3e), these values are: $\sim 22 R_{E}(\mathrm{LQ}), \sim 26 R_{E}(\langle\mathrm{GMD}\rangle)$, and $\sim 30 R_{E}$ (UQ) and, for GML (Fig. 3f), $\sim 18 R_{E}$ (LQ), $\sim 21 R_{E}(\langle\mathrm{GML}\rangle)$, and $\sim 25 R_{E}$ (UQ). Fig. $3 \mathrm{~g}$ indicates that the Alfvénic Mach number distribution shows that most shocks at 1 AU are typically weak, since most events show this number in the interval $1.0<M_{A}<3$.

\subsection{Correlation between GMD and $\theta_{x_{n}}$}

Fig. 4 shows results of correlation analyses between GMD (left column) and GML (right column) with shock impact angle. In order to isolate effects of shock strength, i.e., very weak or very strong shocks, the events are organized in three different shock strength categories represented by shock speeds here defined as follows: weak shocks $\left(300 \mathrm{~km} \mathrm{~s}^{-1}<v_{s}<450 \mathrm{~km} \mathrm{~s}^{-1}\right.$, Fig. 4a), moderate shocks (450 km s $\mathrm{km}^{-1}<v_{s}<600 \mathrm{~km} \mathrm{~s}^{-1}$, Fig. 4b), and strong shocks $\left(600 \mathrm{~km} \mathrm{~s}^{-1}<v_{s}<750 \mathrm{kms}^{-1}\right.$, Fig. 4c). In all panels, the color bars indicate shock speeds whose limits are constrained to the shock speeds of the corresponding shock strength category.

Fig. $4 \mathrm{a}$ and $\mathrm{b}$ show that the weakest shock strength category is the most numerous and clustered category, with GMD values between $10 R_{E}$ and $36 R_{E}$. The average value of GMD in this category is $20.19 R_{E}$. As a general trend of this analysis, GMD values tend to decrease with the increase of $\theta_{x_{n}}$ and $v_{s}$. For shocks with $\theta_{x_{n}}>160^{\circ}$, GMD values assume lower values and almost all shock speeds are larger than $400 \mathrm{~km} \mathrm{~s}^{-1}$. The LQ and UQ values are $16.05 \mathrm{R}_{\mathrm{E}}$ and $23.76 \mathrm{R}_{\mathrm{E}}$, respectively. The correlation coefficient for the category of weak shocks is $\mathrm{R}=-0.60$ for GMD. On the other hand, the correlation of GML with $\theta_{x_{n}}$ is very weak $(\mathrm{R}=-0.06)$ because GML is not aligned
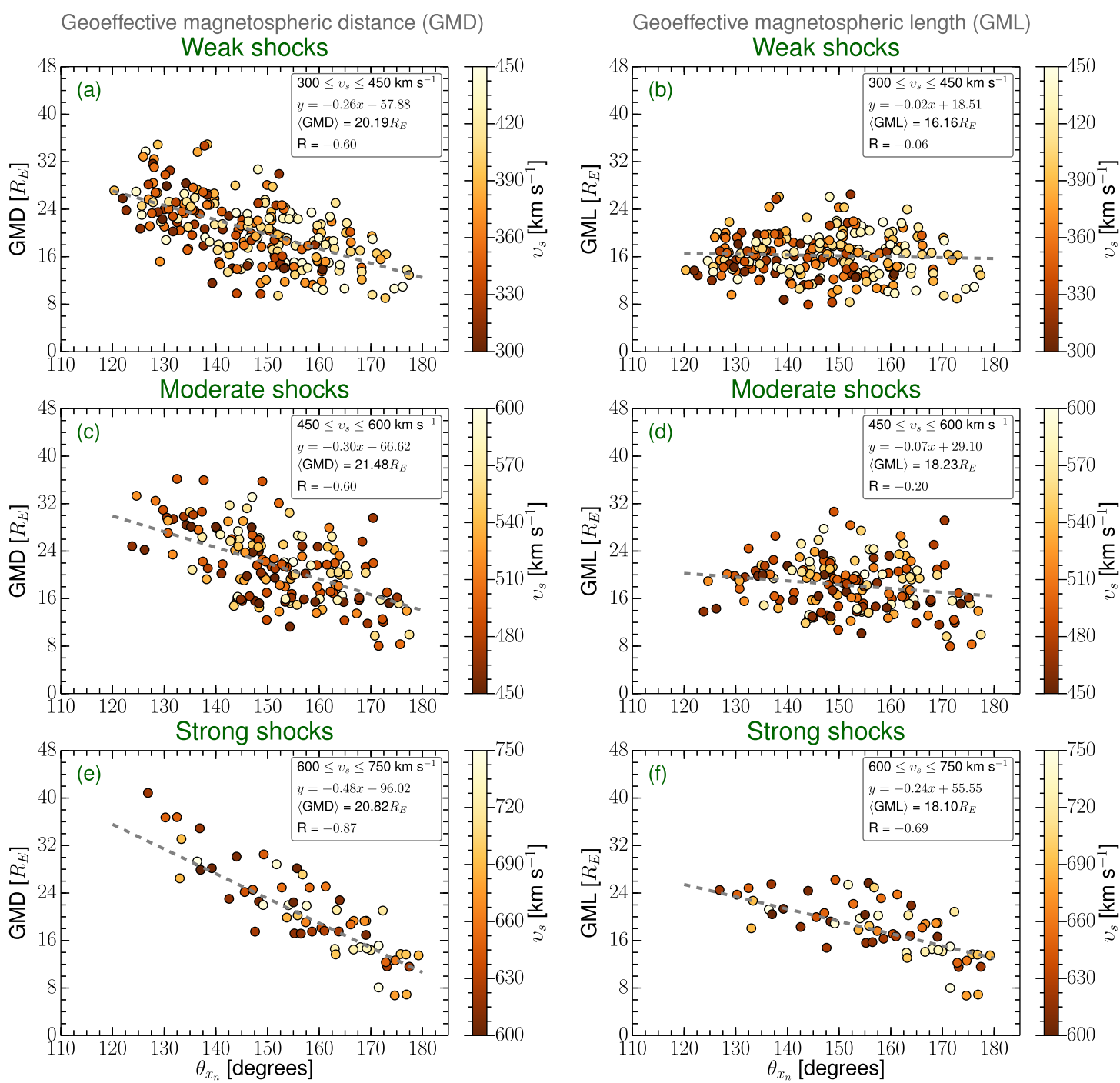

Fig. 4. GMD obtained by the Takeuchi et al. (2002) model plotted as a function pf $\theta_{x_{n}}$. Shocks are arranged in three different categories according to their strength: weak shocks (a), moderate shocks (b), and strong shocks (c). The color bars indicate the shock speed values according to each shock strength category. Correlation coefficients and $\langle\mathrm{GMD}\rangle$ values are shown in each panel. (For interpretation of the references to colour in this figure legend, the reader is referred to the web version of this article.) 
with the direction of shock propagation. All GMLs are constrained between $8 \mathrm{R}_{\mathrm{E}}$ and $26 \mathrm{R}_{\mathrm{E}}$. The GML LQ and $\mathrm{UQ}$ values are $13.62 \mathrm{R}_{\mathrm{E}}$ and $18.64 \mathrm{R}_{\mathrm{E}}$ in this category.

GMD values are more spread in the moderate shock strength category (Fig. 4c), with $9 R_{E}<$ GMD $<37 R_{E}$. Most shocks with $\theta_{x_{n}}<140^{\circ}$ have GMD $>24 R_{E}$. Most shocks with $\theta_{x_{n}}>160^{\circ}$ have GMD $<22 R_{E}$, approximately the mean value in this category but no particular trend is seen in the shock speed values. In comparison to the previous category, small GMD values seem to have been caused by nearly frontal shocks with low speeds in the category. On the other hand, some shocks with speeds from middle values to the maximum value in the category had large GMD values presumably caused by the impact of highly inclined shocks. These results show that the shock impact angles may play a more important role than the shock speeds in determining geoeffective magnetospheric distances. The correlation coefficients for GMD in this category is $\mathrm{R}=-0.60$. The correlation coefficient for GML is $\mathrm{R}=-0.20$, which indicates a low correlation of GML with $\theta_{x_{n}}$ when compared to the GMD correlation coeeficient. GML assume values between $8 \mathrm{R}_{\mathrm{E}}$ and $32 \mathrm{R}_{\mathrm{E}}$. The statistical values for GML in this category are: $15.00 \mathrm{R}_{\mathrm{E}}(\mathrm{LQ})$, $18.23 \mathrm{R}_{\mathrm{E}}(\langle\mathrm{GML}\rangle)$, and $21.00 \mathrm{R}_{\mathrm{E}}(\mathrm{UQ})$.

Finally, the category of strong shocks, Fig. $4 \mathrm{c}$, shows the most clear relationship between shock speed, shock impact angle, and geoeffective magnetospheric distances. Most shocks in this category have $\theta_{x_{n}}>140^{\circ}$. All events with $\theta_{x_{n}}<140^{\circ}$ are associated with GMD values above the average of $\sim 21 R_{E}$, with GMD $>\sim 24 R_{E}$. In this case, all events show $v_{s}<700 \mathrm{~km} \mathrm{~s}^{-1}$. For cases in which $\theta_{x_{n}}>140^{\circ}$, most shocks have shock speeds larger than $\sim 670 \mathrm{~km} \mathrm{~s}^{-1}$. For $\theta_{x_{n}}>160^{\circ}$, most events have GMD $<$ $22 R_{E}$, with shock speeds larger than $680 \mathrm{~km} \mathrm{~s}^{-1}$. The quartiles in this category are $14.83 \mathrm{R}_{\mathrm{E}}(\mathrm{LQ})$ and $24.97 \mathrm{R}_{\mathrm{E}}$ (UQ). The correlation coefficient is $\mathrm{R}=-0.87$. The correlation between GML and $\theta_{x_{n}}$ is stronger than the other correlations in the other categories for the strongest shock category, with $\mathrm{R}=-0.69$. The statistical results are: $\mathrm{LQ}, 14.51 \mathrm{R}_{\mathrm{E}} ;\langle\mathrm{GML}\rangle=18.10 \mathrm{R}_{\mathrm{E}}$; and $\mathrm{UQ}$, $21.26 \mathrm{R}_{\mathrm{E}}$.

By comparing highly inclined shocks in each category, say shocks with $\theta_{x_{n}}<140^{\circ}$, with fixed $\theta_{x_{n}}$, GMD increases with the strength of the category. This indicates that shock speeds are larger for roughly similar RTs.

Fig. 4 shows that the anti-correlation increases with the increase of the shock strength in each category. This anticorrelation is more evident for shocks with small impact angles. In general, these results show that high speed shocks with small impact angles cause $\mathrm{SI}^{+}$events with short RTs and geoeffective distances. Correlation analyses for the RTs performed in the same fashion show the same trends as those shown by GMD and GML. Since very similar results have already been published by Wang et al. (2006) with SYM-H data, we do not reproduce them here. Table 1 summarizes the statistical results obtained for GMD and GML in this study.

\section{Discussion and conclusion}

The first clear association between $\mathrm{SI}^{+} \mathrm{RT}$ and shock inclinations was reported by Takeuchi et al. (2002). They observed an unusually high RT ( $230 \mathrm{~min})$ that ought to have been caused by the impact of an IP shock whose normal performed a large angle with the GSE X line in the equatorial plane. That shock showed a very gradual compression of the magnetotail even though Wind recorded a sharp increase in the solar wind dynamic pressure upstream of the Earth. They attributed that long RT to the fact that the very inclined IP shock had to cover an unusually large distance while compressing the geoeffective magnetosphere. Based on these observations, Takeuchi et al. (2002) introduced the concept of geoeffective magnetopause as a substitution for the concept of magnetic cavity suggested by Nishida (1966), associated with frontal shocks. The main difference between these concepts correspond to the fact that the Takeuchi et al. (2002) model takes the shock impact angle into account for computation of the subsequent geoeffective magnetospheric distances covered by IP shocks.

Guo et al. (2005) modeled the interaction of two IP shocks with the magnetosphere under similar downstream conditions, but with different angles of impact. These authors observed that the two shocks, a frontal shock and an inclined shock, led the magnetosphere-ionosphere system to very similar quasi-steady final states, but the system evolution caused by the impact of the inclined shock took longer to occur. These results were confirmed experimentally by Wang et al. (2006), who performed a statistical analysis of shock impact angle, speed, and $\mathrm{SI}^{+} \mathrm{RT}$ using ACE data. They concluded that fast shocks that hit the magnetosphere almost frontally led to $\mathrm{SI}^{+}$events with short RTs. Similar conclusions were obtained by Selvakumaran et al. (2017).

In this paper, we investigated the effects of IP shock impact angles on the control of geoeffective magnetospheric distances defined during times of magnetospheric compressions associated with $\mathrm{SI}^{+} \mathrm{RT}$ and shock speeds. We calculated the geoeffective magnetospheric distances by using the model introduced by Takeuchi et al. (2002) under the assumption of shock geoeffective time (represented by RT) suggested by Nishida (1966). By taking the comparison between two IP shocks with similar strengths and different inclinations as an example, we showed that the one with high inclination (HIS) took longer to sweep over a larger distance in comparison to the one with small impact angle (NFS). These results agree well with the observation results of Takeuchi et al. (2002) and modeling results of Guo et al. (2005), as well as with the statistical studies of Wang et al. (2006) and Selvakumaran et al. (2017). These examples were used to geometrically illustrate the application of the Takeuchi et al. (2002) model to determine geoeffective magnetospheric distances taking into account the shock impact angle, as indicated by Eqs. (1) and (2) and shown in Fig. 2.

Statistical results derived from the present study for $\mathrm{SI}^{+}$ RTs show that most events have RTs between 4 min and 
Table 1

Summary of statistical results for geoeffective magnetospheric distance (GMD) and geoeffective magnetospheric length (GML) and their correlation results with shock impact angle $\left(\theta_{x_{n}}\right)$, expressed by the correlation coefficient R. GMD results are shown on the left-hand-side of the table, while GML results are shown on the right-hand-side of the table. Lower quartile (LQ) values, mean ( $\langle\mathrm{GMD}\rangle,\langle\mathrm{GML}\rangle)$, and upper quartile (UQ) values are shown for each shock strength category.

\begin{tabular}{cccccc}
\hline & Geoeffective magnetospheric distance & & \multicolumn{2}{c}{ Geoeffective magnetospheric distance } \\
GML $\left.=\left[v_{s} \times \mathrm{RT}\right]\right]\left|\cos \left(\theta_{x_{n}}\right)\right|$
\end{tabular}

$8 \mathrm{~min}$, with $\langle\mathrm{RT}\rangle=6.58 \mathrm{~min}$ (Fig. 3c). These results are similar to the results reported by Maeda et al. (1962). In addition, the mean shock speed velocity is $468.57 \mathrm{~km} \mathrm{~s}^{-1}$ (Fig. 3b). According to Takeuchi et al. (2002), assuming $\theta_{x_{n}}=180^{\circ}$, these results produce GMD (or GML) of $29.04 \mathrm{R}_{\mathrm{E}}$, in remarkable agreement with their prediction of $30 \mathrm{R}_{\mathrm{E}}$. However, results for GMD in the direction of shock propagation show that most shocks are associated with GMD values between $8 R_{E}$ and $40 R_{E}$, with average $\sim 20 \mathrm{R}_{\mathrm{E}}$, while $6 \mathrm{R}_{\mathrm{E}}<\mathrm{GML}<36 \mathrm{R}_{\mathrm{E}}$, with average GML of $\sim 26 R_{E}$. This large GMD $(G M L)$ range indicates a strong impact angle control of the size of the magnetosphere when responding to shock compressions. For example, the minimum GMD $\left(8.4 \mathrm{R}_{\mathrm{E}}\right)$ is obtained for a shock with $\mathrm{RT}=1 \mathrm{~min}, v_{s}=893.2 \mathrm{~km} \mathrm{~s}^{-1}$, and $\theta_{x_{n}}=164^{\circ}$, while the maximum GMD $\left(49.6 \mathrm{R}_{\mathrm{E}}\right)$ is obtained for a shock with $\mathrm{RT}=11 \mathrm{~min}, v_{s}=478.4 \mathrm{~km} \mathrm{~s}^{-1}$, and $\theta_{x_{n}}=146^{\circ}$.

Results of correlation analyses between GMD with $\theta_{x_{n}}$ show that, for nearly frontal shocks $\left(\theta_{x_{n}}>160^{\circ}\right)$, the faster and the more frontal the shock impact, the shorter the geoeffective magnetospheric distances. This result should be expected since the same conditions apply to RTs of $\mathrm{SI}^{+}$ events (Guo et al., 2005; Wang et al., 2006; Selvakumaran et al., 2017). For highly inclined shocks, i.e., $\theta_{x_{n}}<140^{\circ}$, GMD increases with the shock strength category for a fixed $\theta_{x_{n}}$ value. The correlation coefficients for GMD were higher in the category of strong shocks because this distance is calculated along the shock propagation direction which corresponds to the direction in which the most geoeffective compression occurs. The dependence of GMD on $\theta_{x_{n}}$ clearly indicates that there is no intrinsic time or distance of the magnetosphere in response to shocks. GMD depends on shock impact angle and shock speed, which implies that GMD and RT are associated with the sweeping of the magnetosphere by the shock. Correlation coefficients for GML (Eq. (2)) are very low because this distance is not aligned with the shock propagation direction. This result shows that the shock impact angle plays a major role in determining the geoeffectiveness of shocks with different impact angles, confirming previous results (Guo et al., 2005; Wang et al., 2006; Samsonov, 2011; Oliveira and Raeder, 2014; Samsonov et al., 2015; Oliveira and Raeder, 2015; Oliveira et al., 2016; Oliveira, 2017; Selvakumaran et al., 2017; Oliveira and Samsonov, 2018; Oliveira et al., 2018).

In addition, as stated in the introductory section and shown in Fig. 1, other shock parameters such as IP shock $\mathrm{RT}$ and thickness may play a role in controlling $\mathrm{SI}^{+} \mathrm{RT}$, GMD and GML as a response to shock impacts. A future work including these parameters, as well as solar wind and IMF conditions, will be undertaken in a forthcoming work.

Several works have reported on results of coronal mass ejection (CME)/shock arrival time forecasting and prediction through observational and modeling efforts (Zhao and Feng, 2014; Zhao and Dryer, 2014; Tucker-Hood et al., 2015), including tracking of possible radial CME propagation (Mäkelä et al., 2016). However, a clear relationship between $\mathrm{CME} /$ shock impact angle and the subsequent triggering of geomagnetic activity is not currently included in these prediction tools. As a result, we suggest the inclusion of CME/shock impact angles as an important factor of tool capabilities for the improvement and consequently more realistic predictions and forecasting of space weather events.

\section{Acknowledgments}

J.T.R. acknowledges the NAROP (Naval Academy Research Opportunity Program) for the internship opportunity at NASA/GSFC under mentorship of Dr. Denny Oliveira and organization by Dr. Alexa Halford. D.M.O. acknowledges the NASA-SR grants 13-SRITM13 2-0011 393 and HSR-MAG14 2-0062 under contract with UMBC. A.B. acknowledges the support by the NASA Living With a Star Jack Eddy Postdoctoral Fellowship Program, administered by UCAR's Cooperative Programs for the Advancement of Earth System Science (CPAESS). The authors acknowledge the Wind and ACE teams for providing their data through NASA's CDAWeb interface. The authors also greatly acknowledge the SuperMAG PI Dr. Jesper W. Gjerloev and the whole SuperMAG team for 
data availability and the website convenience for data download.

\section{References}

Alves, M.V., Echer, E., Gonzalez, W.D., 2011. Geoeffectiveness of solar wind interplanetary magnetic structures. J. Atmos. Solar Terr. Phys. 73 (11-12), 1380-1384. https://doi.org/10.1016/j.jastp.2010.07.024.

Andréeová, K., Pulkkinen, T.I., Laitinen, T.V., Pr ech, L., 2008. Shock propagation in the magnetosphere: Observations and MHD simulations compared. J. Geophys. Res. 113 (A09224). https://doi.org/ 10.1029/2008JA013350.

Araki, T., 1977. Global structure of geomagnetic sudden commencements. Planet. Space Sci. 25 (4), 373-384. https://doi.org/10.1016/0032-0633 (77)90053-8.

Araki, T., 1994. A physical model of the geomagnetic sudden commencement. In: Engebretson, M.J., Takahashi, K., Scholer, M. (Eds.), Solar Wind Sources of Magnetospheric Ultra-Low-Frequency Waves Geophysical Monograph Series, vol. 81, American Geophysical Union, Washington, DC, pp. 183-200. https://doi.org/10.1029/GM081p0183.

Araki, T., Takeuchi, T., Araki, Y., 2004. Rise time of geomagnetic sudden commencements - statistical analysis of ground geomagnetic data. Earth, Planets Space 56 (2), 289-293. https://doi.org/10.1186/ BF03353411.

Dessler, A.J., Francis, W.E., Parker, E.N., 1960. Geomagnetic storm sudden-commencement rise times. J. Geophys. Res. 65 (9), 2715-2719. https://doi.org/10.1029/JZ065i009p02715.

Gjerloev, J.W., 2009. A global ground-based magnetometer initiative. Eos Trans. AGU 90 (27), 230-231. https://doi.org/10.1029/2009EO270002.

Guo, X.-C., Hu, Y.-Q., Wang, C., 2005. Earth's magnetosphere impinged by interplanetary shocks of different orientations. Chin. Phys. Lett. 22 (12), 3221-3224. https://doi.org/10.1088/0256-307X/22/12/067.

Kaufmann, R.L., Konradi, A., 1969. Explorer 12 magnetopause observations: large-scale nonuniform motion. J. Geophys. Res. 74 (14), 3609-3627. https://doi.org/10.1029/JA074i014p03609.

Kilpua, E.K.J., Lumme, K., Andréeová, E., Isavnin, A., Koskinen, H.E. J., 2015. Properties and drivers of fast interplanetary shocks near the orbit of the Earth (1995-2013). J. Geophys. Res.: Space Phys. 120 (6), 4112-4125. https://doi.org/10.1002/2015JA021138.

Landau, L.D., Lifshitz, E.M., 1960. Electrodynamics of Continuous Media. Pergamon Press, Oxford, England.

Maeda, H., Sakurai, K., Ondoh, T., Yamamoto, M., 1962. A study of solar-terrestrial relationships during the IGY and IGC. Ann. Geophys. $18,305-333$

Mäkelä, P., Gopalswamy, N., Yashiro, S., 2016. The radial speedexpansion speed relation for Earth-directed CMEs. Space Weather 14 (5), 368-378. https://doi.org/10.1002/2015SW001335.

Newell, P.T., Gjerloev, J.W., 2012. SuperMAG-based partial ring current indices. J. Geophys. Res. 117 (A05215), 1-15. https://doi.org/10.1029/ 2012JA017586.

Nishida, A., 1966. Interpretation of SSC rise time. Report Ionosphere Space Res. Japan 20, 42-44.

Nishida, A., Jacobs, J.A., 1962. Equatorial enhancement of world-wide changes. J. Geophys. Res. 67 (12), 4937-4940. https://doi.org/10.1029/ JZ067i012p04937.

Nopper, R.W., Hughes, W.J., Maclennan, C.G., McPherron, R.L., 1982. Impulse-excited pulsations during the July 29, 1977, event. J. Geophys. Res. 87 (A8), 5911-5916. https://doi.org/10.1029/JA087iA08p05911.

Oliveira, D.M., 2017. Magnetohydrodynamic shocks in the interplanetary space: a theoretical review. Braz. J. Phys. 47 (1), 81-95. https://doi.org/ 10.1007/s13538-016-0472-x.

Oliveira, D.M., Raeder, J., 2014. Impact angle control of interplanetary shock geoeffectiveness. J. Geophys. Res.: Space Phys. 119 (10), 81888201. https://doi.org/10.1002/2014JA020275.

Oliveira, D.M., Raeder, J., 2015. Impact angle control of interplanetary shock geoeffectiveness: a statistical study. J. Geophys. Res.: Space Phys. 120 (6), 4313-4323. https://doi.org/10.1002/2015JA021147.
Oliveira, D.M., Samsonov, A.A., 2018. Geoeffectiveness of interplanetary shocks controlled by impact angles: a review. Adv. Space Res. 61 (1), 1-44. https://doi.org/10.1016/j.asr.2017.10.006.

Oliveira, D.M., Raeder, J., Tsurutani, B.T., Gjerloev, J.W., 2016. Effects of interplanetary shock inclinations on nightside auroral power intensity. Braz. J. Phys. 46 (1), 97-104. https://doi.org/10.1007/ s13538-015-0389-9.

Oliveira, D.M., Arel, D., Raeder, J., Zesta, E., Ngwira, C.M., Carter, B. A., Yizengaw, E., Halford, A.J., Tsurutani, B.T., Gjerloev, J.W., 2018 Geomagnetically induced currents caused by interplanetary shocks with different impact angles and speeds. Space Weather 16 (6), 636647. https://doi.org/10.1029/2018SW001880.

Ondoh, T., 1963. Longitudinal distribution of ssc rise time. J. Geomagn. Geoelectr. 14 (4), 198-207. https://doi.org/10.5636/jgg.14.198.

Pesnell, W.D., 2015. Predictions of solar cycle 24: how are we doing? Space Weather 14 (1), 10-21. https://doi.org/10.1002/2015SW001304.

Samsonov, A.A., 2011. Propagation of inclined interplanetary shock through the magnetosheath. J. Atmos. Solar Terr. Phys. 73 (1), 1-9. https://doi.org/10.1016/j.jastp.2009.10.014.

Samsonov, A.A., Sergeev, V.A., Kuznetsova, M.M., Sibeck, D.G., 2015. Asymmetric magnetospheric compressions and expansions in response to impact of inclined interplanetary shock. Geophys. Res. Lett. 42 (12), 4716-4722. https://doi.org/10.1002/2015GL064294.

Selvakumaran, R., Veenadhari, B., Ebihara, Y., Kumar, S., Prasad, D.S V.V.D., 2017. The role of interplanetary shock orientation on SC/SI rise time and geoeffectiveness. Adv. Space Res. 59 (5), 1425-1434. https://doi.org/10.1016/j.asr.2016.12.010.

Shue, J.-H., Song, P., Russell, C.T., Steinberg, J.T., Chao, J.K., Zastenker, G., Vaisberg, O.L., Kokubun, S., Singer, H.J., Detman, T.R., Kawano, H., 1998. Magnetopause location under extreme solar wind conditions. J. Geophys. Res. 103 (A8). https://doi.org/10.1029/ 98JA01103, 17,691-17,70.

Singh, A., Rathore, V.S., Singh, R.P., Singh, A.K., 2017. Source identification of moderate $(-100 \mathrm{nT}<$ Dst $<-50 \mathrm{nT})$ and intense geomagnetic storms (Dst $<-100 \mathrm{nT}$ ) during ascending phase of solar cycle 24. Adv. Space Res. 59 (1), 209-1222. https://doi.org/10.1016/j. asr.2016.12.006.

Takeuchi, T., Russell, C.T., Araki, T., 2002. Effect of the orientation of interplanetary shock on the geomagnetic sudden commencement. J. Geophys. Res. 107 (A12). https://doi.org/10.1029/2002JA009597, SMP 6-1-SMP 6-10.

Tsurutani, B.T., Lakhina, G.S., Verkhoglyadova, O.P., Gonzalez, W.D., Echer, E., Guarnieri, F.L., 2011. A review of interplanetary discontinuities and their geomagnetic effects. J. Atmos. Solar Terr. Phys. 73 (1), 5-19. https://doi.org/10.1016/j.jastp.2010.04.001.

Tucker-Hood, K., Scott, C., Owens, M., Jackson, D., Barnard, L., Davies, J.A., Crothers, S., Lintott, C., Simpson, R., Savani, N.P., Wilkinson, J., Harder, B., Eriksson, G.M., L Baeten, E.M., Wan Wah, L.L., 2015. Validation of a priori CME arrival predictions made using real-time heliospheric imager observations. Space Weather 13 (1), 35-48. https:// doi.org/10.1002/2014SW001106.

Wang, C., Li, C.X., Huang, Z.H., Richardson, J.D., 2006. Effect of interplanetary shock strengths and orientations on storm sudden commencement rise times. Geophys. Res. Lett. 33 (L14104), 1-3. https://doi.org/10.1029/2006GL025966.

Wilken, B., Goertz, C.K., Baker, D.N., Higbie, P.R., Fritz, T.A., 1977. The SSC on July 29, 1977 and its propagation within the magnetosphere. J. Geophys. Res 87 (A8), 5901-5910. https://doi.org/10.1029/ JA087iA08p05901.

Zhao, X., Dryer, M., 2014. Current status of CME/shock arrival time prediction. Space Weather 12 (7), 448-469. https://doi.org/10.1002/ 2014SW001060.

Zhao, X.H., Feng, X.S., 2014. Shock Propagation Model version 2 and its application in predicting the arrivals at Earth of interplanetary shocks during Solar Cycle 23. J. Geophys. Res.: Space Phys. 119 (1), 1-10. https://doi.org/10.1002/2012JA018503. 